INPLASY

PROTOCOL

To cite: Wang et al.

Association between climate conditions and osteoarthritis: a

systematic review and metaanalysis. Inplasy protocol

202130053. doi:

10.37766/inplasy2021.3.0053

Received: 15 March 2021

Published: 16 March 2021

Corresponding author:

Wang Lin

798494395@qq.com

Author Affiliation:

Shanghai University of TCM

Support: 81373665, 81973874.

Review Stage at time of this submission: Formal screening of search results against

eligibility criteria.

Conflicts of interest:

None declared.

\section{Association between climate conditions and osteoarthritis: a systematic review and meta-analysis}

*Wang, L1; *Xu, Q2; Chen, Y3; Wang, X4; Jiang, D5; Tan, Z6; Li, M7; Shen, $\mathrm{X}^{8} ; \mathrm{Cao}, \mathrm{Y}^{9}$.

Review question / Objective: To investigate the association between weather conditions and the incidence, progression of osteoarthritis, respectively, as well as the relative efficacy of different weather conditions(e.g. wind, temperature, relative humidity, barometric pressure, etc.) for the progression of OA symptomatic and structural changes.

Information sources: Systematic literature searches were undertaken using EMBASE, MEDLINE ,Web of Science, PubMed and the Cochrane Library. The subject terms were used for separate retrievals, and the Boolean operators $\backslash " O R \backslash "$, and \"AND\" were used to connect the terms. Developed a search strategy for related documents from publication to November 2020. To acquire more feasible studies besides the database searches, we will hand search the reference lists of all involved studies, as well as other relevant review articles, current evidence in order to prevent to miss the relative additional researches by electronic database searching. We will search for npublished and ongoing study at (www.who.int/trialsearch and ClinicalTrials.gov) using the object I"the relation between weather condition (climates) and OAl".

*Wang Lin and Xu Qinguang contributed equally.

INPLASY registration number: This protocol was registered with the International Platform of Registered Systematic Review and Meta-Analysis Protocols (INPLASY) on 16 March 2021 and was last updated on 16 March 2021 (registration number INPLASY202130053).

\section{INTRODUCTION}

Review question / Objective: To investigate the association between weather conditions and the incidence, progression of osteoarthritis, respectively, as well as the relative efficacy of different weather conditions(e.g. wind, temperature, relative 
humidity, barometric pressure, etc.) for the progression of $O A$ symptomatic and structural changes.

Condition being studied: Osteoarthritis (OA) is a disease caused by multiple factors?frequently accompanied by joint pain, stiffness, and limited mobility, which seriously affect the daily life and health of patients. Related studies report that about 360 million people worldwide suffer from OA. Among people over 60 years old, the incidence rate is $10 \%$ for men and $18 \%$ for women . It is extremely susceptible to the changes of season or weather conditions. Many studies have reported that high humidity and low temperature often induce osteoarthritis or aggravate symptoms. However, There is not enough research to prove the inevitability of the connection between climate and osteoarthritis, the conclusions of individual case-control studies, cohort studies, or cross-sectional surveys are relatively one-sided, with low credibility, insufficient and the credibility is low. Therefore, in order to accurately understand the correlation between climate factors and osteoarthritis, Metaanalysis is a more comprehensive and accurate better choice. Through the results from meta-analysis of climate factors and osteoarthritis can further guide doctors $\backslash^{\prime}$ clinical practice and patients to avoid and reduce the impact on them in time. Bayesian network meta-analysis was used to estimate the relative effect size (ES) and corresponding $95 \%$ credibility interval (Crl)

\section{METHODS}

Participant or population: We included studies that examined the relationship between certain weather conditions and osteoarthritis.

Intervention: Exposure(s): Weather conditions, eg:wind, temperature, humidity, barometric pressure, and other common weather conditions.

Comparator: Different degrees of weather conditions (eg:the highest humidity and lowest temperature classification).
Study designs to be included: Include all case-control studies, cohort studies or cross-sectional studies, focusing on the relationship between different weather conditions (temperature, wind, humidity, air pressure, and other common weather conditions) and osteoarthritis.

Eligibility criteria: Inclusion criteria: (1) patients with osteoarthritis, (2) observational study including case-control study, cohort study or cross-sectional study, (3) researches focuses on the relationship between weather conditions and osteoarthritis (incidence, progress, prognosis) (4) data provided in the research results or can be converted into OR value, $95 \% \mathrm{Cl}$ and standard error (SE) (5) researches is published in English. Exclusion criteria: (1) among the irrelevant population, (2) not an observational study, (3) no relevant exposure, (4) The results of the research are irrelevant, (5) agreement or notes, review literature, or summary without data, (6) duplicate publications, documents in the same area in the same year.

Information sources: Systematic literature searches were undertaken using EMBASE , MEDLINE ,Web of Science, PubMed and the Cochrane Library. The subject terms were used for separate retrievals, and the Boolean operators \"OR\", and \"AND\" were used to connect the terms. Developed a search strategy for related documents from publication to November 2020. To acquire more feasible studies besides the database searches, we will hand search the reference lists of all involved studies, as well as other relevant review articles, current evidence in order to prevent to miss the relative additional researches by electronic database searching. We will search for npublished and ongoing study at (www.who.int/trialsearch and ClinicalTrials.gov) using the object I"the relation between weather condition (climates) and OAl.

Main outcome(s): Incidence of osteoarthritis in specific weather conditions, Changes in symptoms of osteoarthritis in specific weather 
conditions, or progression and prognosis of osteoarthritis in specific weather conditions.

Additional outcome(s): $95 \% \mathrm{Cl}$, standard error (SE),or datas can be converted into OR value.

Data management: The two authors WL and XQG will independently extract the following data from the acceptable and feasible observation studies using a standardize form: 1.study design; 2.publication year and authors; 3. trial population (e.g. sample size, age, percentages of people with moderate and severe TBI in the sample); 4. number of subjects, sex, age; 5 . exposures (weather conditions: wind, temperature, relative humidity, barometric pressure, precipitation and sunshine etc.) 6. selected outcomes, the Western Ontario and McMasters University Osteoarthritis Index (WOMAC) or Visual Analogue Scale (VAS) in specific weather conditions. Pain exacerbation of patients with osteoarthritis of the Pain Numeric Rating Scale (NRS 0e10). The evaluation of functional capacity of joint (Health Assessment Questionnaire, HAQ) in relative weather conditions. the Odds ratios (ORs), relative risks (RRs), hazard ratios (HRs), respective 95\% confidence intervals (CIs), correlation coefficients, regression coefficients. We will also extract data on stiffness and function of patients with osteoarthritis under certain weather conditions, changes in joint structure and bone and joint imaging. standard error (SE) or dates can be converted into OR value, the observation methods, frequency and handling of missing data, and selective reporting of outcomes. Given that our cognitive level with the document, we will not ignore any significance data of each study so that the extracting data be accuracy and comprehensive. During this process, for any divergences, we will resolve them by discussing and consulting If we fail to get an agreement, a third review author should attend to.

Quality assessment / Risk of bias analysis: The Newcastle-Ottawa Scale (NOS) items will be used to ascertain the quality of the included studies by two independent reviewers and consensus will be reached in case of disagreement.

Strategy of data synthesis: We will preferably select the valid effect size measure (Odds Ratio) or standard error (SE). The FEM (Fixed Effect Model) or REM (Random Effects Model) combination method will be chosen based on the methodological similarities between included researches. The combined effect size will be plotted by a forest plot.

Subgroup analysis: If possible, a subgroup analysis will be conducted to explore the relationship between specific weather conditions and the incident of osteoarthritis, explore the relationship between specific weather conditions and osteoarthritis symptoms, explore the relationship between specific weather conditions and the progression of osteoarthritis, and explore specific weather The relationship between the condition and the prognosis of osteoarthritis.

Sensitivity analysis: Where possible the comparisons deemed to be at high risk of bias, we will perform sensitivity analyses to assess the reliability of the overall studies by deleting the inspection high risk of bias studies. We will then analyses the studies we deem to be low risk of bias again to qualitative overall results.

Country(ies) involved: China.

Keywords: Association; Climate Conditions; Osteoarthritis; Review.

Contributions of each author:

Author 1 - Wang Lin.

Email: 798494395@qq.com

Author 2 - Xu Qinguang.

Email: xuqinguang1608@126.com

Author 3 - Chen Yan.

Author 4 - Wang Xuezong.

Author 5 - Jiang Ding.

Author 6 - Tan Zecheng.

Author 7 - Li Ming.

Author 8 - Shen Xue.

Author 9 - Cao Yuelong. 